\title{
Efficient bioconversion of raspberry ketone in Escherichia coli using fatty acids feedstocks
}

Chen Chang ${ }^{1,2+}$, Bo Liu ${ }^{2 \dagger}$, Yihong Bao ${ }^{1,4^{*}}$, Yong Tao $^{2,3^{*}}$ and Weifeng Liu $2,3^{*}$ (D)

\begin{abstract}
Background: Phenylpropanoid including raspberry ketone, is a kind of important natural plant product and widely used in pharmaceuticals, chemicals, cosmetics, and healthcare products. Bioproduction of phenylpropanoid in Escherichia coli and other microbial cell factories is an attractive approach considering the low phenylpropanoid contents in plants. However, it is usually difficult to produce high titer phenylpropanoid production when fermentation using glucose as carbon source. Developing novel bioprocess using alternative sources might provide a solution to this problem. In this study, typical phenylpropanoid raspberry ketone was used as the target product to develop a biosynthesis pathway for phenylpropanoid production from fatty acids, a promising alternative low-cost feedstock.

Results: A raspberry ketone biosynthesis module was developed and optimized by introducing 4-coumarate-CoA ligase (4CL), benzalacetone synthase (BAS), and raspberry ketone reductase (RZS) in Escherichia coli strains CR1-CR4. Then strain CR5 was developed by introducing raspberry ketone biosynthesis module into a fatty acids-utilization chassis FA09 to achieve production of raspberry ketone from fatty acids feedstock. However, the production of raspberry ketone was still limited by the low biomass and unable to substantiate whole-cell bioconversion process. Thus, a process by coordinately using fatty-acids and glycerol was developed. In addition, we systematically screened and optimized fatty acids-response promoters. The optimized promoter Pfrd3 was then successfully used for the efficient expression of key enzymes of raspberry ketone biosynthesis module during bioconversion from fatty acids. The final engineered strain CR8 could efficiently produce raspberry ketone repeatedly using bioconversion from fatty acids feedstock strategy, and was able to produce raspberry ketone to a concentration of $180.94 \mathrm{mg} / \mathrm{L}$ from soybean oil in a 1-L fermentation process.

Conclusion: Metabolically engineered Escherichia coli strains were successfully developed for raspberry ketone production from fatty acids using several strategies, including optimization of bioconversion process and fine-tuning key enzyme expression. This study provides an essential reference to establish the low-cost biological manufacture of phenylpropanoids compounds.
\end{abstract}

Keywords: Raspberry ketone, Fatty acids feedstock, Bioconversion, Phenylpropanoids, Escherichia coli

*Correspondence: baoyihong@163.com; taoyong@im.ac.cn; liuwfv@im.ac.cn

${ }^{\dagger}$ Chen Chang and Bo Liu contributed equally to this work

${ }^{1}$ College of Forestry, Northeast Forestry University, No. 26 Hexing Road, Harbin, Heilongjiang Province 150040, PR China

${ }^{2}$ CAS Key Laboratory of Microbial Physiological and Metabolic Engineering, State Key Laboratory of Microbial Resources, Institute of Microbiology, Chinese Academy of Sciences, NO. 1 Beichen West Road, Chaoyang District, Beijing 100101, PR China

Full list of author information is available at the end of the article

\section{Background}

Major industrial biotechnological effort is focused on developing efficient bioproduction routes to high value-added chemicals that are more cost-effective than conventional petrochemical routes $[1,2]$. Therefore, increasing important bioprocess metrics, such as titer, yield of target products, and using cheaper raw materials, have become central to metabolic engineering $[3,4]$. Phenylpropanoids, including flavonoids, are a diverse 
family of compounds mainly synthesized by plants from aromatic amino acids phenylalanine and tyrosine [5]. Phenylpropanoids are among the most important natural products and possess diverse important functions with applications in various fields, such as pharmaceuticals, food, cosmetics, and healthcare products [6-8]. However, the low phenylpropanoid contents in plants limit the use of plant extraction routes for production $[9,10]$. Recently, phenylpropanoid production by microbial cell factories has attracted significant attention [11, 12].

Typical phenylpropanoid molecules contain an aromatic phenyl group, and their biosynthesis usually requires coumaroyl-coenzyme A (CoA) and several molecules of malonyl-CoA as precursors. Raspberry ketone (RK; 4-(4-hydroxyphenyl)-2-butanone) is the simplest phenylpropanoid and has been wildly used in the fields of cosmetics, food additive. Biosynthesis of raspberry ketone requires one coumaroyl-coenzyme $\mathrm{A}(\mathrm{CoA})$ and one malonyl-CoA as precursors. In previous studies, metabolic engineering for raspberry ketone and other phenylpropanoids productions, such as naringenin and curcumin, has been conducted in E. coli, yeast, and other microbial factories [13-15]. Major approach to metabolic engineering for raspberry ketone and other phenylpropanoids production involves introducing key enzyme responses to target metabolic pathways. 4-Coumaroyl-CoA is generated from 4-coumaric acid under catalysis by 4-coumaroyl CoA ligase (4CL) [16]. 4-Coumaroyl-CoA is then condensed with several malonyl-CoA extender units under catalysis by chalcone synthase (CHS) to yield chalcone. Chalcone, under catalysis by various enzymes, includes isomerases, hydroxylases, oxidoreductases. And post-modification enzymes, such as glycosyltransferases and methyltransferases, and acyltransferases, are then transformed into different phenylpropanoid products [17].

However, the development of industrial-scale processes for phenylpropanoid production is still facing serious problems. However, no applicable process for high production metrics have been established.The fermentation of some phenylpropanoids often requires complex culture conditions and processes to improve product titers $[18,19]$. During the fermentation process of some phenylpropanoids such as raspberry ketone, it is difficult to develop an applicable bioconversion process to enrich biomass [20, 21]. It is also reported that the production of some phenylpropanoids is significantly reduced in glucose media $[22,23]$. This makes fermentation procedures significantly more difficult and expensive when translated to large-scale processing.

Fatty acids (FAs) can serve as ideal alternative biomass resources with several advantages. Important metabolic precursor acetyl-CoA can be obtained from fatty acids through $\beta$-oxidation without carbon loss. This allows several products to be produced with high theoretical yields. Furthermore, the $\beta$-oxidation of fatty acids can release a large amount of reducing power essential for the synthesis of target products [24, 25]. Fatty acids materials can be obtained from various low-cost industrial and domestic wastes and industrial byproducts [26]. For example, when obtained from palm industry byproducts, the average carbon price per ton of palmitic acid is about $\$ 35$ per ton of $C$ (calculated from $\$ 570$ per ton of palmitic acid extract), which is much lower than the average carbon price for glucose ( $\$ 46$ per ton of $C$, calculated from $\$ 275$ per ton of glucose) [27]. In previous studies, we have successfully developed efficient routes to produce target chemicals, such as 3-hydroxypropionic acid and lycopene, in high yields from fatty acids $[28,29]$.

In this study, we sought to develop an efficient route for phenylpropanoid production using fatty acids as the feedstock. Typical phenylpropanoid raspberry ketone was used as the target product to develop a biosynthesis pathway for phenylpropanoid production from fatty acids. The pathway from fatty acids to phenylpropanoids has several advantages. Fatty acids could generate malonyl-CoA precursors for raspberry ketone biosynthesis with higher theoretical yield than glucose (Fig. 1). Bioproduction of raspberry ketone from fatty acids also might provide a solution to the bioprocess problem when using glucose feedstock. In this study, an efficient bioconversion process for raspberry ketone production was developed. Furthermore, to facilitate heterogeneous gene regulation and develop an ideal fermentation process, we systematically constructed a promoter system for use under fatty acids conditions. This study, for the first time, developed an efficient tools and protocols for phenylpropanoid production via fatty acids feedstock routes.

\section{Results}

\section{Development and optimization of raspberry ketone biosynthesis module}

First, 4-coumarate-CoA ligase (4CL), benzalacetone synthase (BAS), and raspberry ketone reductase (RZS) were introduced and overexpressed in $E$. coli strains to develop a raspberry ketone biosynthesis module. Genes of At4CL1 from Arabidopsis thaliana, RpBAS from Rheum palmatum, and RiRZS1 from Rubus idaeus [21] were code optimized and constructed into either medium-copy-number p15A-derived plasmids or low-copy-number r6k-derived plasmids. Different plasmid combinations were transformed into $E$. coli BW25113. After one-step fermentation using complex enriched medium (CM medium, see materials and methods) for $24 \mathrm{~h}$, strain CR1 containing pLB1a-RB and PYB1s-4CL could produce raspberry ketone with a concentration of $1.25 \pm 0.1 \mathrm{mg} / \mathrm{L}$, while strain CR2 

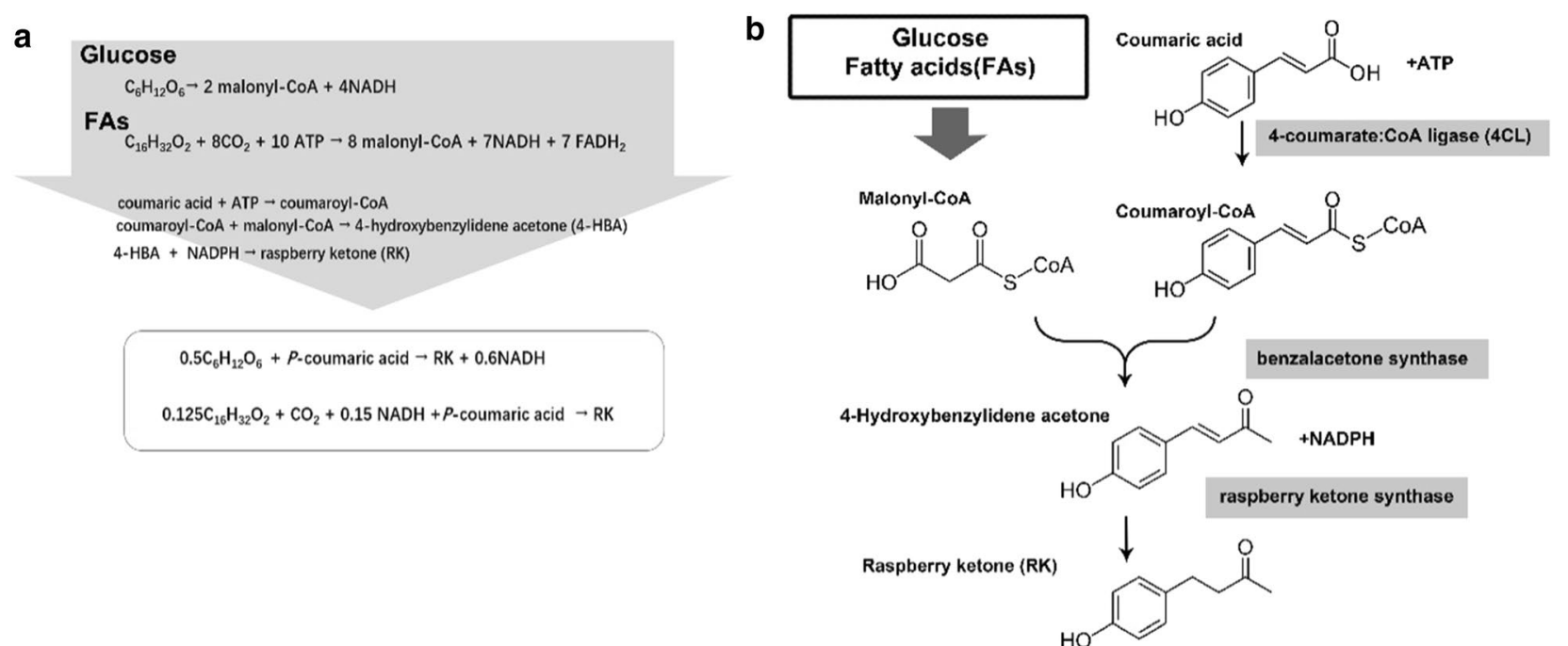

Fig. 1 Production route of raspberry ketone (RK) from glucose, and fatty acids (FAs). a Equations from different carbon sources to RK; equations in the box show overall theoretical stoichiometry. $\mathbf{b}$ Enzymes involved in RK biosynthesis pathway

containing pLB1a-4CL and pYB1s-RB could produce a raspberry ketone concentration of $1.03 \pm 0.1 \mathrm{mg} / \mathrm{L}$. In contrast, when RiRZS1, RpBAS, and At4CL1 were co-expressed in a single low-copy-number r6k-derived plasmid (pLB1a-RB4, strain CR3), Raspberry ketone production was not detected. This result implied that AT4CL1 expression was a key factor influencing raspberry ketone production. Consistent with these results, further replacing the r6k-derived plasmid with a medium-copy-number p15A-derived plasmid (pYB1s-RB4, strain CR4) resulted in a raspberry ketone concentration of $13.1 \pm 0.2 \mathrm{mg} / \mathrm{L}$ (Fig. 2b).

Raspberry ketone production of CR4 was then carried out with different fermentation time periods. It was shown CR4 could produce raspberry ketone with $28.3 \pm 2.5 \mathrm{mg} / \mathrm{L}$ after fermentation for $48 \mathrm{~h}$ in $\mathrm{CM}$ medium. However, only $11.1 \pm 2.5 \mathrm{mg} / \mathrm{L}$ raspberry ketone was produced in glucose medium (48 h) (Fig. 2c). The strategy of whole-cell bioconversion using harvested cells is usually associated with high production titers. Thus, a two-step bioconversion experiment was carried out: cells enrichment and protein induction were carried out in CM medium first. Then cells were harvested and resuspended for bioconversion for the production of raspberry ketone. However, the raspberry ketone titers were very low when using bioconversion strategy in both $\mathrm{CM}$ medium $(5.2 \pm 0.2 \mathrm{mg} / \mathrm{L})$ and glucose $(0 \mathrm{mg} / \mathrm{L})$ (Fig. $2 \mathrm{~d})$. It was shown $p$-coumaric acid was hardly consumed during bioconversion. This implied the biosynthesis pathway of raspberry ketone was not active in this condition.

\section{Development strains for raspberry ketone production from fatty acids feedstock}

The raspberry ketone biosynthesis module was then transformed into a fatty acids-utilization host to develop the fatty acids route for raspberry ketone production. The degradation process of fatty acids is generally carried out by $\beta$-oxidation pathway. Taking palmitic acid as an example, long-chain fatty acids enter the cells through outer membrane transport protein $(\mathrm{FadL})$ and are then activated to fatty acylCoA by fatty acyl-CoA synthetase(FadD). After entering the mitochondrial matrix, it undergoes multiple cycles under the catalysis of fatty acid $\beta$-oxidase system, and acetyl-CoA and fatty acyl-CoA are produced in each cycle. Acyl-CoA produces acetyl-CoA under the action of acyl-CoA dehydrogenase (FadE) and ketoacyl-CoA thiolase (FadAB). The pYB1s-RB4 plasmid was introduced into fatty acids utilization chassis cell FA09 to develop strain CR5 [28, 30-32]. First, one-step fermentation strategy was used to produce raspberry ketone using fatty acids as feedstock. It was shown that the titer of raspberry ketone was about $21.5 \pm 2.4 \mathrm{mg} / \mathrm{L}$ after fermentation for $48 \mathrm{~h}$, which was a little lower than that produced in CM medium. However, the titers were still found to be very low when the whole-cell bioconversion strategy was used (Fig. 3a). To assess the fermentation process in a different medium, the biomass of strains was recorded. It was shown that the biomass of strains during fermentation in fatty acids was much lower than in both CM medium and glucose (Fig. 3b). This result indicated 


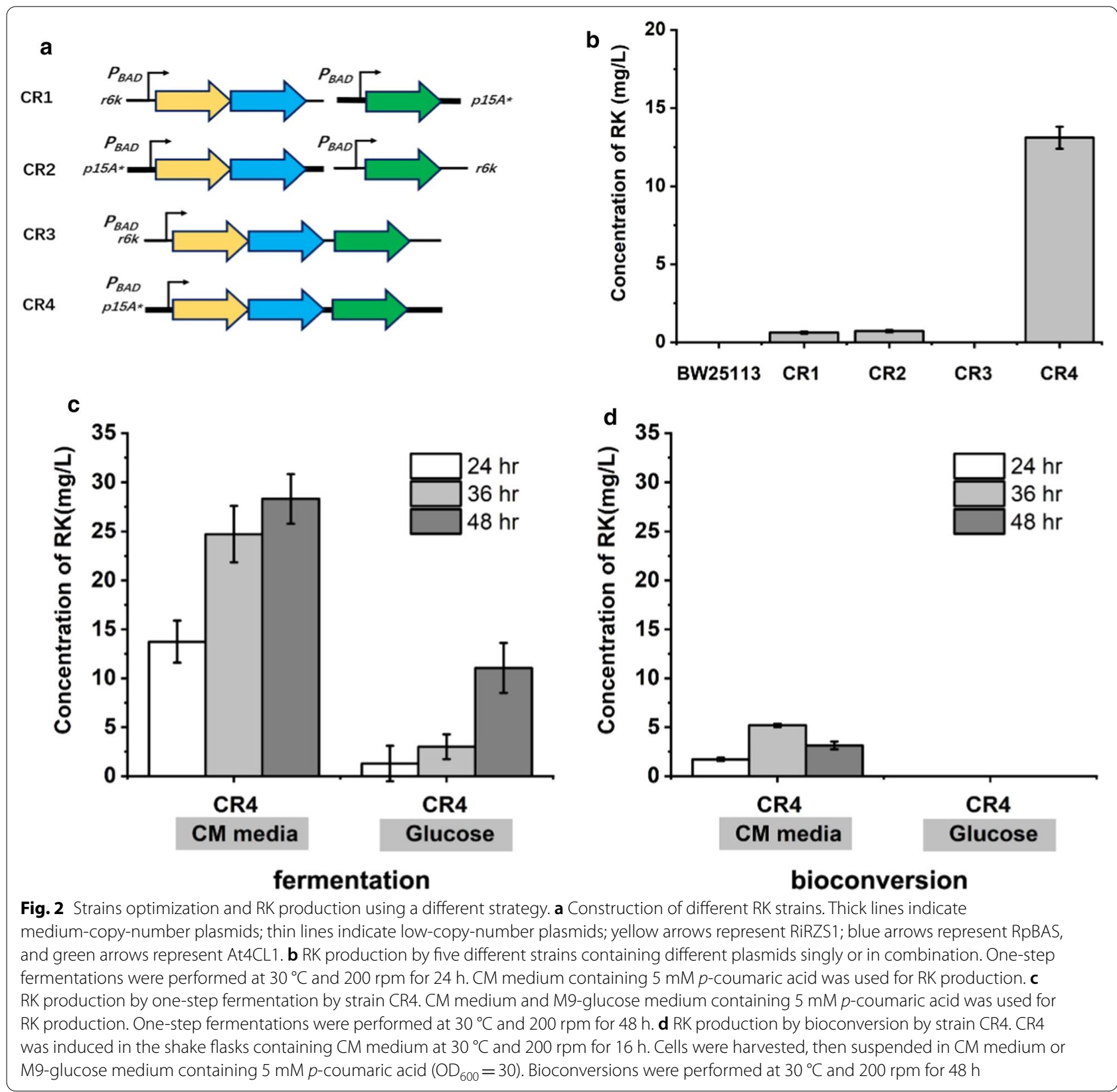

that the fermentation conditions in the fatty acids medium should be optimized.

\section{Efficient raspberry ketone production by condition optimization}

Next, we sought to enhance cell growth and performance during fermentation in fatty acids. The fermentation medium was optimized by added different fatty acids compositions in the modified CM medium (MCM medium). The results indicated the biomass of all strains were similar to that in CM medium (about
$\left.\mathrm{OD}_{600}=9-11\right)$. Raspberry ketone titers of strain CR5 gradually increased with increasing concentrations of fatty acids and reached $41.5 \pm 2.0 \mathrm{mg} / \mathrm{L}$ in $1 \%$ fatty acids after fermentation for $24 \mathrm{~h}$ (Fig. 4f2-f4). Additional 1\% glucose significantly decreased raspberry ketone titers to $13.7 \pm 2.9 \mathrm{mg} / \mathrm{L}$ (Fig. $4 \mathrm{f5}$ ). On the contrary, an additional $0.5 \%$ and $1 \%$ glycerol further increased raspberry ketone titers to $44.4 \pm 3.5$ and $51.3 \pm 7.3 \mathrm{mg} / \mathrm{L}$ (named as MCM7 medium), respectively (Fig. 4f6-f7). Nevertheless, the optimized fatty acids fermentation medium was also suitable for the bioconversion strategy. Cultured 

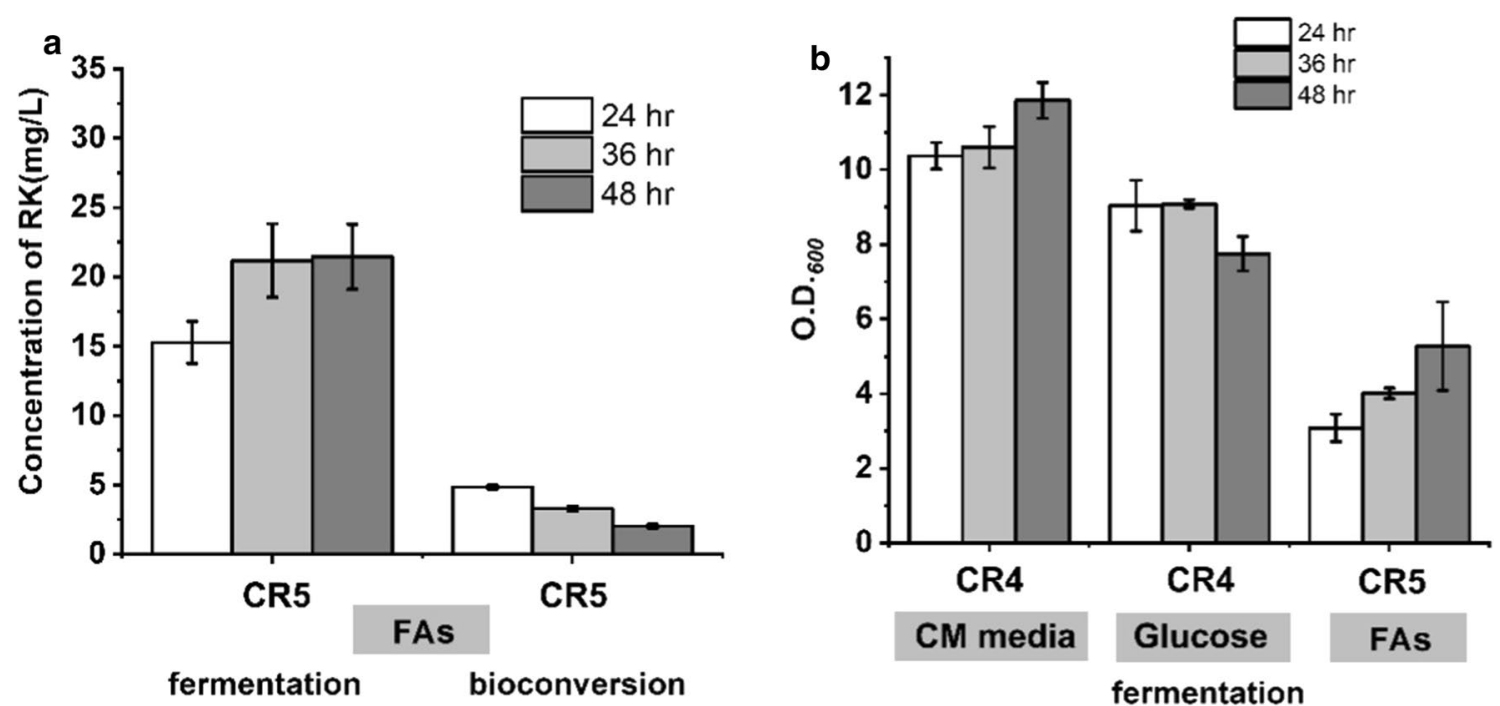

Fig. 3 a RK production by CR5 strain in FAs medium using both fermentation and bioconversion strategy. M9-FAs medium containing 5 mM p-coumaric acid was used for fermentation. CM medium was used for bioconversion. CR5 was induced first in CM medium and harvested, then suspended in CM-FAs medium containing $5 \mathrm{mM}$ p-coumaric acid $\left(\mathrm{OD}_{600}=30\right)$. Fermentations and bioconversion were performed at $30^{\circ} \mathrm{C}$ and $200 \mathrm{rpm}$. b Comparison of biomass in the different fermentation medium. CM medium, M9-glucose, and M9-FAs medium containing $5 \mathrm{mM}$ p-coumaric acid were used for fermentation. Fermentations were performed at $30^{\circ} \mathrm{C}$ and $200 \mathrm{rpm}$ for $48 \mathrm{~h}$

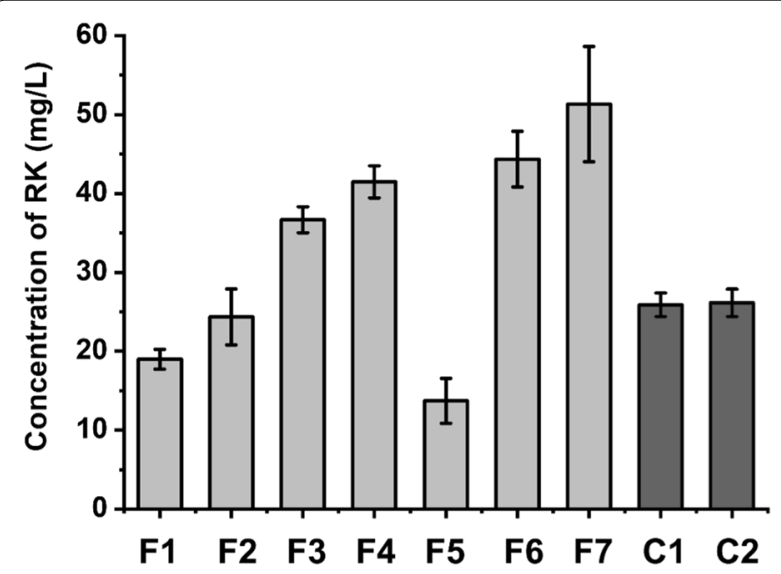

Fig. 4 RK production under different fermentation conditions for CR5. MCM medium containing $5 \mathrm{mM}$ p-coumaric acid was used for F1-F7 fermentation. The concentrations of different compositions used were: F1: none; F2: 0.2\% FA; F3: 0.5\% FA; F4:1\% FA; F5: 1\%FA + 0.5\%glucose; F6: 1\%FA + 0.5\% glycerol; F7: 1\%FA + 1\% glycerol. Fermentations were performed at $30^{\circ} \mathrm{C}$ and $200 \mathrm{rpm}$ for 24 h. MCM7 medium was used for C1 and C2 bioconversion. C1: cells were induced by MCM7 medium and harvested, then suspended in MCM7 medium containing $5 \mathrm{mM}$-coumaric acid $\left(\mathrm{OD}_{600}=30\right)$; C2: Cells from CR5 cultured in MCM7 medium were harvested and suspended in MCM7 medium $\left(\mathrm{OD}_{600}=30\right)$. Bioconversions were performed at $30^{\circ} \mathrm{C}$ and $200 \mathrm{rpm}$ for $24 \mathrm{~h}$

cells of CR5 strain were resuspended in MCM7 medium with a starting $\mathrm{OD}_{600}=30$, and bioconversion was then carried out. Raspberry ketone titer of CR5 could reach
$25.9 \pm 1.5 \mathrm{mg} / \mathrm{L}$ after bioconversion for $24 \mathrm{~h}$ (Fig. 4c1). A similar titer $(26.1 \pm 1.8 \mathrm{mg} / \mathrm{L})$ was also obtained when using cells harvested from CR5 cultured in MCM7 medium (Fig. 4c2).

\section{Screening and optimizing fatty acids response promoters}

According to above results, raspberry ketone titer was significantly increased by both fermentation and bioconversion in the optimized FA medium. This indicated fatty acids medium provided necessary factors related to raspberry ketone biosynthesis pathways. Considering that the expression of key enzymes, such as AT4CL1, are key limit factors for raspberry ketone production, expression of these enzymes should be fine regulated in response to the change of condition. Therefore, expression under promoters induced by fatty acids compositions provides a superior strategy to control the expression of key pathway enzymes. Then, we conducted promoter mining work to screen candidate promoters in response to fatty acids condition. We mainly focused on promoters involved in TCA cycles. This was because the expression of genes within TCA cycle was significantly enhances in fatty acids condition [28]. TCA-related promoters were constructed using green fluorescent protein (GFP) as reporter. The GFP intensity of different promoters was then analyzed after culture in fatty acids medium. Under several TCA-related promoters, GFP intensity increased in fatty acids conditions (data not shown). It was notable that the GFP intensity significantly increased under FA 


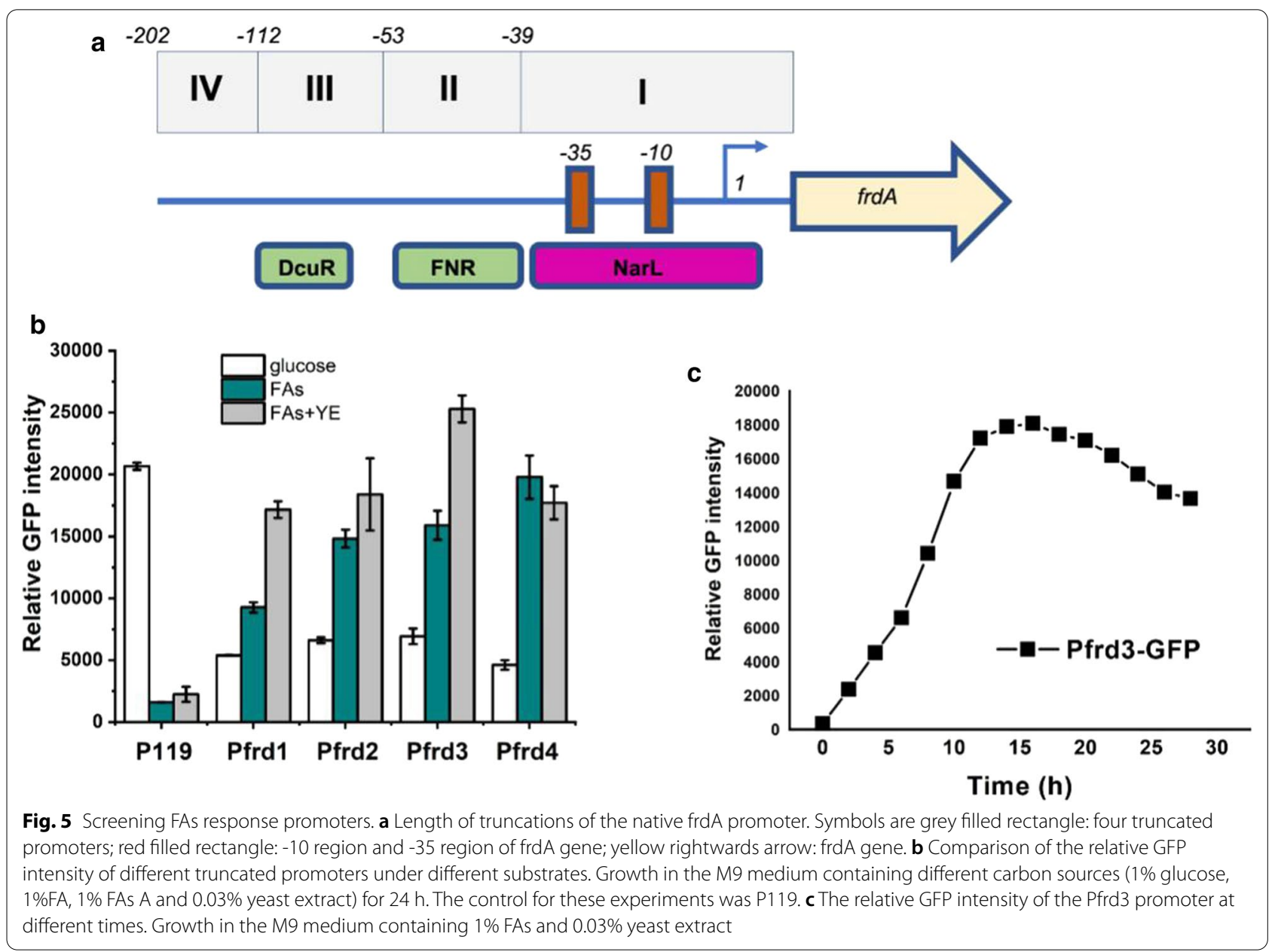

conditions using promoters PfrdA. Then four promoters with different truncations were designed (Fig. 5a). Different conditions were then investigated to determine the influence on the induction profiles of these promoters (Fig. 5c). The results indicated that GFP intensity using all promoters was significantly increased in fatty acids condition. Furthermore, additional 0.03\% YE could further improve the GFP expression. The peak of GFP intensity occurred about $13 \mathrm{~h}$ after cultured in fatty acids condition.

\section{Modifying different modules using FA-induced promoters}

Then we carried out genetic modification using above promoters to develop strains that were suitable for bioconversion strategy. First, an additional copy of the AT4CL1 gene was expressed under the control of Pfrd3 and chromosomally inserted at the poxB site, resulting in new strain CR6. Raspberry ketone production by CR6 was about $57.7 \pm 1.7 \mathrm{mg} / \mathrm{L}$ after bioconversion for $24 \mathrm{~h}$, which was much higher than that by CR5 (31.3 $\pm 1.5 \mathrm{mg} / \mathrm{L})$ (Fig. 6). In E. coli, Type II fatty acid synthase (FAS) was responsible for FA production from acetyl-CoA and subsequent elongation using malonylacyl carrier protein (ACP) [33]. We attempted to increase the intracellular malonyl-CoA level by disrupting the competitive pathways. The fatty acids biosynthesis pathway is a major pathway for malonyl-CoA consumption. Thus, $f a b B$ gene encoding $\beta$-ketoacyl-[acyl carrier protein] synthases (fabB) that catalyze the condensation reactions of long-chain fatty acid synthesis should be knocked-out. Meanwile, studies have also been shown that the introduction of cyanobacteria carbon concentration mechanism (CCM) in E. coli could increase the concentration of inorganic carbon in the cell and further increase the malonyl-CoA supply, improved the production of 3- hydroxypropionic acid effectively [9, 27]. Therefore, the bicarbonate transporter (BT) gene from Synechococcus sp. and the carbonic anhydrase (CA) gene from Anabaena sp. PCC 7120 were fused and chromosomal expressed by inserting at fabB site under the control of Pfrd3 promoter, obtained the strain CR7, and the final RK concentration reached $65.5 \pm 2.0 \mathrm{mg} / \mathrm{L}$. 

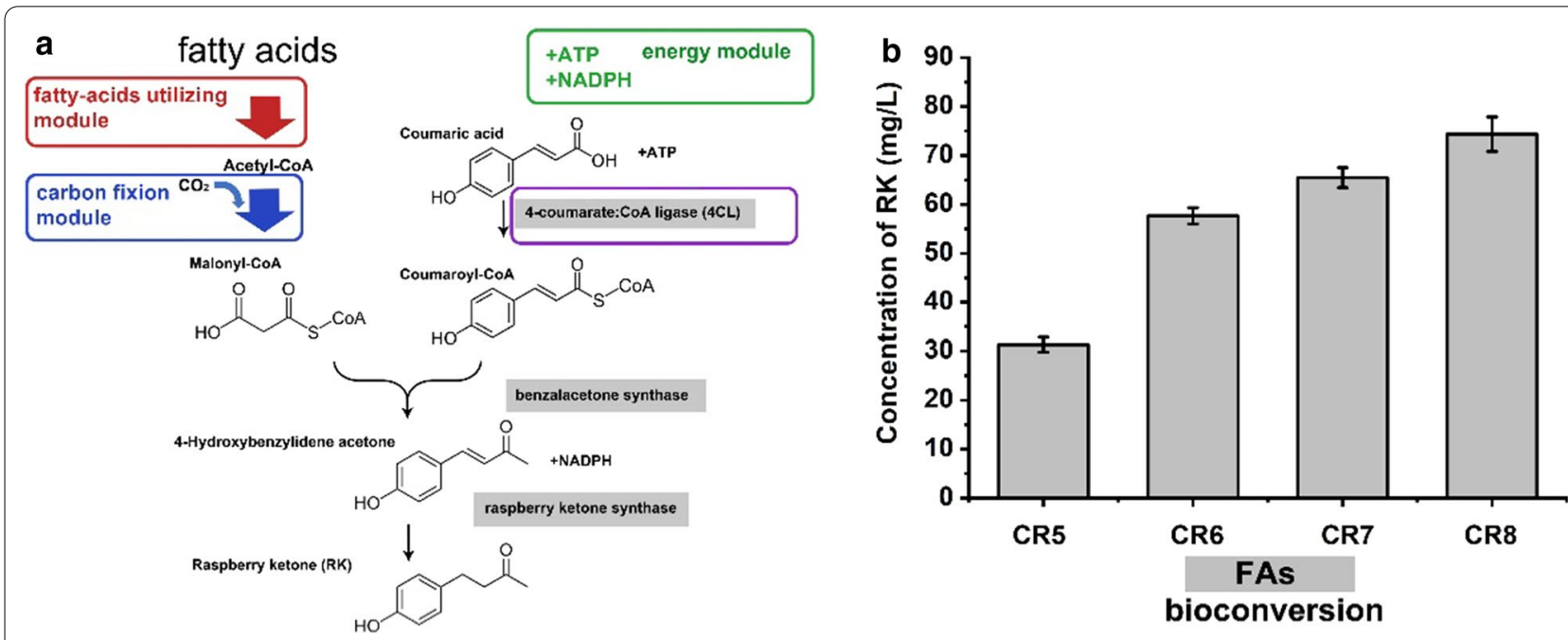

Fig. 6 Metabolic engineering for RK production from FAs and RK production by different strains. a RK biosynthesis pathway from FAs; $\mathbf{b}$ RK production by different strains. Bioconversions were performed at $30^{\circ} \mathrm{C}$ and $200 \mathrm{rpm}$. A palmitic acid concentration of $1 \%$ was used for RK production

Furthermore, RK reductase (RiRZS1) was NADPHdependent, and the $\beta$-oxidation of fatty acids produces a large amount of NADH [34]. We attempted to further accumulate RK by regulating the conversion of NADH to NADPH. The tetramer $[\mathrm{PntB}]_{2}[\mathrm{PntA}]_{2}$ encoded by pntAB, the complex was a membrane-bound pyridine nucleotide transhydrogenase, which has a higher affinity for NADH and a weaker affinity for NADPH, so the enzyme mainly catalyzed the conversion of NADH into NADPH. Thus, additional pntAB under the regulation of Pfrd3 promoter was introduced to obtain strain CR8, which further increased the yield by $20 \%$ to $74.4 \pm 3.5 \mathrm{mg} / \mathrm{L}$, showing that NADPH had a considerable influence on RK synthesis.

\section{Efficient production of raspberry ketone by repeat bioconversion process}

The bioconversion conditions were further optimized to achieve higher raspberry ketone production titers. Biomass during bioconversion was investigated at 10,20,30, and 40 O.D. It was shown the higher titer was obtained at 30 O.D. biomass (Additional file 1: Fig. S2). Then the speed of rotation was further optimized. Raspberry ketone titer with $93.3 \pm 2.4 \mathrm{mg} / \mathrm{L}$ could be observed at an optimized condition, $220 \mathrm{rpm}, 30$ O.D biomass, bioconversion for $30 \mathrm{~h}$.

Based on CR8, a continual fermentation process was conducted. First, CR8 strain was cultured and fermentation in MCM7 medium for $24 \mathrm{~h}$, during which about $126.4 \mathrm{mg} / \mathrm{L}$ raspberry ketone could be produced. Then the cells were collected by centrifugation, resuspended in the MCM7 medium at 30 O.D. After bioconversion for additional $24 \mathrm{~h}, 76.4 \mathrm{mg} / \mathrm{L}$ raspberry ketone could be produced. The above operation was repeated three times. The raspberry ketone titers were 46.1 and $31.4 \mathrm{mg} / \mathrm{L}$ for the second and third bioconversion, respectively (Fig. 7a).

Furthermore, bioconversion was carried out in a $1-\mathrm{L}$ bioreactor. RK production of strain CR8 was investigated by feeding with soybean oil as a single substrate (Fig. 7b and Additional file 1: Fig. S3). CR8 strain was first cultivated in CD medium for $24 \mathrm{~h}$. Then, soybean oil was fed in batches at $1 \mathrm{~g} / \mathrm{L} / \mathrm{h}$. Soybean oil and glycerol were used as mixed substrates and fed according to a 1:1 carbon ratio, the total carbon of the mixed feed was remained the same as the glycerol feed. P-coumaric acid was fed in batches at $2.5 \mathrm{mmol} / \mathrm{L} / 12 \mathrm{~h}$ The final RK concentration was about $203 \mathrm{mg} / \mathrm{L}$ when using mixed substrates reached (Fig. $7 \mathrm{~b}$ ).

\section{Discussion}

Phenylpropanoids, such as resveratrol and naringenin, are a class of natural products widely found in plants and microorganisms, and have important applications in medicines, cosmetics, and health products [6]. To date, no practical industrial process for the microbial production of phenylpropanoids have been developed. Wholecell bioconversion is a more efficient technique route for production of not only raspberry ketone and other phenylpropanoids, but also other diverse bioproducts. However, it is difficult to develop an efficient bioconversion process for raspberry ketone production previously. Raspberry ketone and other phenylpropanoids synthesis require $p$-coumaric acid or cinnamic acid as precursor, which are derived from aromatic amino acids. After 

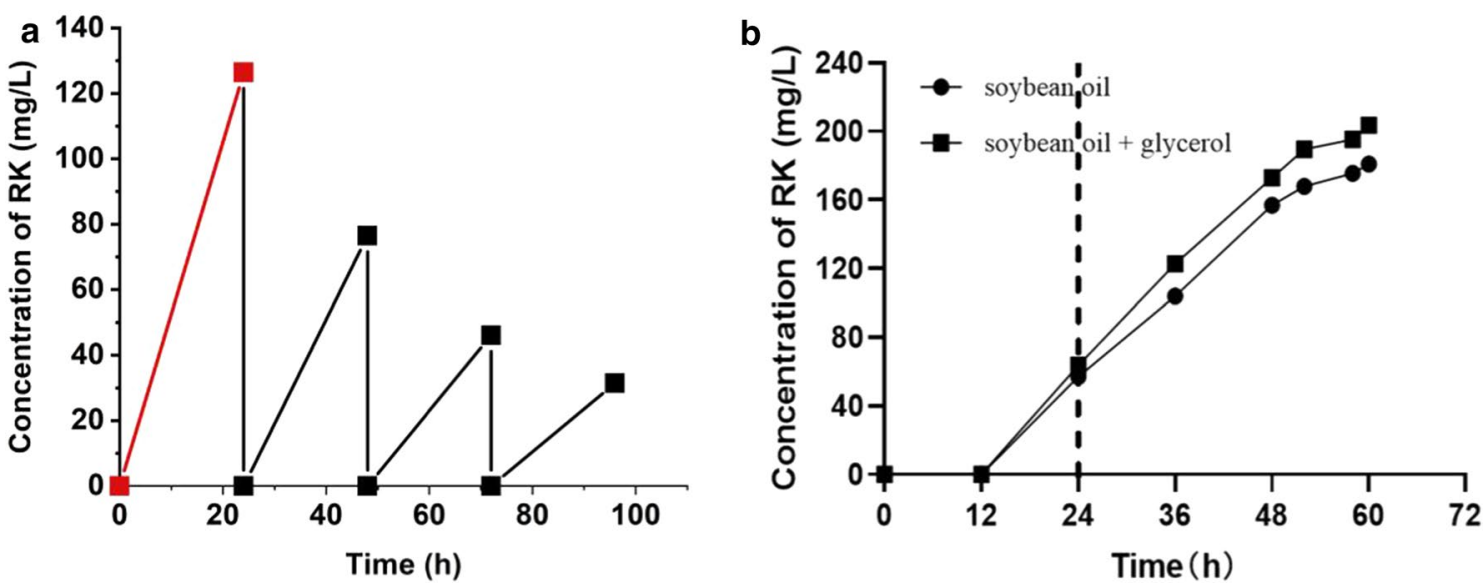

Fig. 7 A. RK production by repeat bioconversion process. Red line indicates induction and cell growth stage. Bioconversion were performed at $30^{\circ} \mathrm{C}$ and $220 \mathrm{rpm}$. A palmitic acid concentration of $1 \%$ was used for RK production. B. Fed-batch bioconversion in 1-L bioreactors for RK production using soybean oil or soybean oil + glycerol as substrate. The left side of the dotted line represents the cell growth and protein induction phase

activation by CoA, $p$-coumaric acid or cinnamic acid is polymerized with different units of malonyl-CoA to form the final target product. However, similar to previous reports, our study found that 4CL-mediated activation of $p$-coumaric acid was the critical rate-limiting step [21]. To our surprise, when glucose was used as the raw material, the raspberry ketone yield was low. Similar phenomenon is also reported in the production of some other phenylpropanoids $[21,35]$. The underling mechanism of the glucose inhibitory effect on bioproduction is still to be explored. There might be several reasons for this. First, the function or expression of key enzymes is limited under glucose conditions. Second, the level of some important factors or metabolites is not sufficient for the biosynthsis pathway. The precursor $p$-coumaric acid is significantly accumulated when glucose was used, suggesting that fine regulation of $4 \mathrm{CL}$ expression and CoA levels in cells was a key factor in this synthesis. To remove this bottleneck and achieve efficient expression of enzyme 4CL in fatty acids raw materials, we systematically screened promoters that can be induced by fatty acids. The results showed that relevant promoters could be used in our strains to successfully increase the raspberry ketone production.

Malonyl-CoA is among the most important metabolites, and can serve as a basic building block for fatty acids biosynthesis. Therefore, malonyl-CoA is the key precursor of diverse fatty-acid-derived compounds, including biofuels [36]. Malonyl-CoA is also a precursor for the microbial synthesis of many pharmaceutically interesting polyketides and natural products, such as phenylpropanoids [37]. Fatty acids are a promising resource for the biosynthesis of malonyl-CoA via carboxylation of acetyl-CoA, which can be efficiently obtained from the $\beta$-oxidation of fatty acids. As fatty acids are an easily obtained feedstock, they are ideal raw materials for novel production routes to natural products [28]. In this study, a novel biosynthesis strategy to produce raspberry ketone (a representative phenylpropanoid) from fatty acids raw materials was reported for the first time. These results demonstrate a novel technical route for the production of similar compounds.

Furthermore, establishing an efficient induction and fermentation process might be key to achieving a highlevel fermentation strategy for phenylpropanoid production. We solved this problem through cooperative utilization of multiple carbon sources and fine-tuning of key enzyme expression. Further studies will focus on using different FA resources as raw materials. In summary, we conducted systematic metabolic engineering of $E$. coli strains to produce raspberry ketone from fatty acids. A module for raspberry ketone synthesis was constructed by introducing 4CL, BAS, and RZS. Then the raspberry ketone synthesis module was combined with the fatty acids utilization module to construct the engineered strain for raspberry ketone production from fatty acids. We systematically screened the fatty acidinducible promoters. The expression of key enzymes under Pfrd3 promoter contributed to $p$-coumaric acid activation, increasing the supply of precursors and NADPH. Finally, the strain CR08 produced raspberry ketone by using multiple carbon sources, and produced $180.94 \mathrm{mg} / \mathrm{L}$ raspberry ketone by using soybean oil in a 1-L fermentation. This study will help use cheaper raw 
materials to produce not only raspberry ketone, but also other important flavonoid compounds.

\section{Materials and methods Strains and reagents}

DNA polymerases, Taq Mix, T4 DNA ligase, Gibson Assembly ${ }^{\circledR}$ One Step Cloning Kit were purchased from Vazyme Biotech Co. Ltd. (Nanjing, China). Restriction enzymes were purchased from New England Biolabs (USA). Plasmid Mini Kits, PCR Clean-Up Kits, and Gel Extraction kits were all obtained from Omega Bio-Tek Co. Ltd. (USA). P-coumaric acid and raspberry ketone was purchased from Solarbio Biochemical Co. Ltd. (Shanghai, China). All other regular chemicals were purchased from ShengGong Biochemical Co. Ltd. (Shanghai, China). The primer synthesis and gene sequencing were done in GENEWIZ, Inc. (Beijing, China).

The E. coli strain DH5 $\alpha$ was used for plasmids construction, BW25113/F' $\mathrm{F}^{\prime}$ were used for protein expression and raspberry ketone production, respectively. Luria-Bertani (LB) medium was used for all molecular construction experiments and strain culture. Strains containing temperature-sensitive plasmids pKD46, pCP20, and pSB1s-cre were cultured at $30{ }^{\circ} \mathrm{C}$, and the remaining strains were cultured at $37^{\circ} \mathrm{C}$ without special instructions.

Luria-Bertani (LB) medium containing $10 \mathrm{~g} / \mathrm{L}$ tryptone, $5 \mathrm{~g} / \mathrm{L}$ yeast extract, and $10 \mathrm{~g} / \mathrm{L} \mathrm{NaCl}$ was used to grow $E$. coli cells unless otherwise stated. $1 \times \mathrm{M} 9$ salts containing $12.8 \mathrm{~g} / \mathrm{L} \quad \mathrm{Na}_{2} \mathrm{HPO}_{4} \cdot 7 \mathrm{H}_{2} \mathrm{O}, 3 \mathrm{~g} / \mathrm{L} \quad \mathrm{KH}_{2} \mathrm{PO}_{4}$, $0.5 \mathrm{~g} / \mathrm{L} \mathrm{NaCl}, 1 \mathrm{~g} / \mathrm{L} \mathrm{NH}_{4} \mathrm{Cl}$ was used to bioconversion. M9-FAs medium containing $1 \times \mathrm{M} 9$ salts, $2 \mathrm{mM} \mathrm{MgSO}_{4}$, $10 \mathrm{~g} / \mathrm{L}$ palmitic acid, M9-FAs + YE (supplemented with YE), M9-glucose (10/L glucose instead of palmitic acid) were used to screen FA response promoters. CM medium containing $10 \mathrm{~g} / \mathrm{L}$ tryptone, $5 \mathrm{~g} / \mathrm{L}$ yeast extract, $5 \mathrm{~g} / \mathrm{L}$ glycerol, $0.5 \mathrm{~g} / \mathrm{L}$ glucose, $25 \mathrm{mM} \mathrm{Na}_{2} \mathrm{HPO}_{4}, 25 \mathrm{mM}$ $\mathrm{KH}_{2} \mathrm{PO}_{4}, 50 \mathrm{mM} \mathrm{NH} \mathrm{Cl}_{4}, 5 \mathrm{mM} \mathrm{Na}_{2} \mathrm{SO}_{4}, 2 \mathrm{mM} \mathrm{MgSO}_{4}$ and trace elements was used for one-step fermentation and cell preparation for bioconversion. Modified CM medium containing $1 \times \mathrm{M} 9$ salts, $5 \mathrm{~g} / \mathrm{L}$ tryptone, $2 \mathrm{mM}$ $\mathrm{MgSO}_{4}$ and trace elements was used to one-step fermentation and bioconversion. When necessary, the antibiotics were used (ampicillin, $100 \mu \mathrm{g} / \mathrm{mL}$; streptomycin, $50 \mu \mathrm{g} / \mathrm{mL}$; and kanamycin, $50 \mu \mathrm{g} / \mathrm{mL}$ ).

\section{Construction of plasmids and strains}

All strains and plasmids used in this study are shown in Table 1. All primers are listed in Additional file 2: Table S1. At4CL1 (GenBank ID: AAA82888.1) from Arabidopsis thaliana, RpBAS (GenBank ID:
AAK82824.1) from Rheum palmatum, and RiRZS1 (GenBank ID: JN166691) from Rubus idaeus were codon-optimized for E. coli expression [17, 38-40].

Molecular cloning and genetic editing were performed using standard protocols. For knockout genes, a single knockout library stored in the laboratory was used to achieve integration through P1 phage infection [41]. pCP20 eliminated the resistance marker by identifying the FRT sites at both ends of the resistance marker gene. The plasmids pYB1s and pLB1a were previously constructed in our laboratory; the specific features were as follows: streptomycin and kanamycin resistance genes, araBAD promoter, multiple cloning sites, rrnB terminator, p15A, and R6k. Promoter replacement and gene insertion replacement used the gene-editing tool plasmids constructed in our laboratory as templates for amplification to obtain homologous recombination fragments. pSB1s-cre eliminated the resistance marker by identifying the lox66 and lox71 sites at both ends of the resistance marker gene.

\section{One-step fermentation conditions}

When used CM medium for one-step fermentation, the recombinant strains were grown in $\mathrm{CM}$ medium to an $\mathrm{OD}_{600}$ of $0.4-0.6$ at $30{ }^{\circ} \mathrm{C}$, added $5 \mathrm{mM} p$-coumaric acid, and $2 \mathrm{~g} / \mathrm{L} \mathrm{L}$-arabinose, then cultured for a given time. When used glucose for one-step fermentation, the recombinant strains were grown in $\mathrm{M} 9$ modified medium to an $\mathrm{OD}_{600}$ of $0.4-0.6$ at $30^{\circ} \mathrm{C}$, added $2 \mathrm{~g} / \mathrm{L} \mathrm{L}$-arabinose and induced overnight, supplemented with $10 \mathrm{~g} / \mathrm{L}$ glucose, $5 \mathrm{mM} p$-coumaric acid, then cultured for a given time. When used fatty acids for one-step fermentation, the process was the same as the glucose for one-step fermentation process, except for supplementing $10 \mathrm{~g} / \mathrm{L}$ fatty acids.

For condition optimization, the following methods were used to optimize the substrate: (1) different concentrations of fatty acids were added (2) $1 \%$ fatty acids and different concentrations of glycerol were used together with different concentrations of fatty acids (2) $1 \%$ fatty acids and $0.5 \%$ glucose.

\section{Bioconversion conditions}

The induction conditions we changed according to the experiment in the study, cells were collected after induction by centrifugation at $6000 \times g$ for $10 \mathrm{~min}$, washed twice with $0.85 \% \mathrm{NaCl}$ solution, suspended in $3 \mathrm{~mL}$ bioconversion medium in a test tube containing $5 \mathrm{mM}$ $p$-coumaric acid and different feedstocks, starting biomass of $\mathrm{OD}_{600}=30$ (unless otherwise specified), growing at $30{ }^{\circ} \mathrm{C}, 200 \mathrm{rpm}$ for $24 \mathrm{~h}$. The bioconversion medium 
Table 1 Strains and plasmids used in this study

\begin{tabular}{|c|c|c|}
\hline Strain & Genotype & Source \\
\hline E. coli BW25113/F' & rrnBT14 $\triangle$ lacZWJ16 hsdR514 $\triangle a r a B A D A H 33 \triangle$ rhaBADLD78 [F' proAB laclaZDM15 Tn10 (Tetr)] & CGSC \\
\hline FA09 & E. coli BW25113/F', $\triangle$ fadR, $\mathrm{P}_{\mathrm{CPA} 1}-$ fadD, $\mathrm{P}_{119}$-fadL, $\triangle$ sthA, $\mathrm{P}_{\mathrm{CPA} 1}-$ pntAB & {$[28]$} \\
\hline CC1 & FA09, $\triangle p 0 \times B:: P_{\text {frd3 }}-A T 4 C L 1$ & This study \\
\hline CC2 & $C C 1, \triangle f a b B:: P_{119-B T}$ BTA & This study \\
\hline CC3 & $C C 2, P_{\text {frd } 3}-p n t A B$ & This study \\
\hline CR1 & E. coli BW25113/F' carrying pLB1a-RB and pYB1s-4CL & This study \\
\hline CR2 & E. coli BW25113/F carrying pYB1s-RB and pLB1a-4CL & This study \\
\hline CR3 & E. coli BW25113/F' carrying pLB1a-RB4 & This study \\
\hline CR4 & E. coli BW25113/F' carrying pYB1s-RB4 & This study \\
\hline CR5 & FA09 carrying pYB1s-RB4 & This study \\
\hline CR6 & CC1 carrying pYB1s-RB4 & This study \\
\hline CR7 & CC2 carrying pYB1s-RB4 & This study \\
\hline CR8 & CC3 carrying pYB1s-RB4 & This study \\
\hline Plasmid & Description & Source \\
\hline pLB1a & araBAD promoter, R6k ori, Ampr & Our laboratory \\
\hline pYB1s & araBAD promoter, p15A ori, Strr & Our laboratory \\
\hline pKD46 & Temperature-sensitive vector carrying Red recombinase, Amp ${ }^{r}$ & {$[42]$} \\
\hline pSB1s-Cre & Temperature-sensitive vector carrying Cre recombinase, Strr & Our laboratory \\
\hline pCP20 & Temperature-sensitive vector carrying FLP recombinase, Amp ${ }^{r}$ & [42] \\
\hline pLB1a-RB & pLB1a containing Rubus idaeus RZS1 gene and Rheum palmatum BAS gene & This study \\
\hline pLB1a-4CL & pLB1a containing Arabidopsis thaliana 4CL1 gene & This study \\
\hline pYB1s-RB & pYB1s containing Rubus idaeus RZS1 gene and Rheum palmatum BAS gene & This study \\
\hline pYB1s- 4CL & pYB1s containing Arabidopsis thaliana 4CL1 gene & This study \\
\hline pLB1a-RB4 & $\begin{array}{l}\text { pLB1a containing Rubus idaeus RZS1 gene, Rheum palmatum BAS gene and Arabidopsis thali- } \\
\text { ana } 4 \text { CL1 gene }\end{array}$ & This study \\
\hline pYB1s-RB4 & $\begin{array}{l}\text { pYB1s containing Rubus idaeus RZS1 gene, Rheum palmatum BAS gene and Arabidopsis thali- } \\
\text { ana } 4 \text { CL1 gene }\end{array}$ & This study \\
\hline pYfrd11s-GFP & frd1 promoter, p15A ori, Str', GFP gene & This study \\
\hline pYfrd21s-GFP & frd2 promoter, p15A ori, Strr', GFP gene & This study \\
\hline pYfrd31s-GFP & frd3 promoter, p15A ori, Str', GFP gene & This study \\
\hline pYfrd41s-GFP & frd4 promoter, p15A ori, Strr', GFP gene & This study \\
\hline
\end{tabular}

changed based on experimental conditions. The carbon sources glucose, glycerol, or fatty acids was also added based on the experimental conditions. In the process of optimizing the bioconversion conditions, the bioconversion starting biomass of $\mathrm{OD}_{600}$ was investigated at 10,20 , 30 and 40 .

\section{Cell growth and fluorescence analysis}

To compare the strength of FA response promoters, the GFP gene was placed under different promoters' control and introduced into a FA utilization chassis cell FA09. After overnight cultured, inoculated to M9-FAs medium, M9-glucose medium, and M9-FAs + YE medium, starting biomass of $\mathrm{OD}_{600}=0.1,37{ }^{\circ} \mathrm{C}$, and $200 \mathrm{rpm}$ culture for $36 \mathrm{~h}$. GFP intensity was measured using the BioTek Synergy Mx enzyme marker (BioTek, Winooski, VT,
USA). Excitation at $468 \mathrm{~nm}$, emission at $512 \mathrm{~nm}$, automatic gain.

Fatty acids emulsified in the medium were opaque emulsion, so $\mathrm{OD}_{600}$ could not be used directly to detect biomass. We mixed an equal volume of ethyl acetate with the bacterial solution, centrifugation at $6000 \times \mathrm{g}$ for $1 \mathrm{~min}$, the fatty acid was sandwiched between the ethyl acetate and the medium, discarded all the supernatant, resuspended the cells with an appropriate amount of $0.85 \% \mathrm{NaCl}$ solution, and monitored the biomass in $\mathrm{OD}_{600}$.

\section{Analytical methods}

HPLC determined product concentration. A $500 \mu \mathrm{L}$ sample was taken and mixed thoroughly with the same amount of absolute ethanol by vortexing $30 \mathrm{~s}$. The sample 
was centrifuged at $8000 \times g$ for $10 \mathrm{~min}$ and filtered with a $0.2 \mu \mathrm{m}$ PES membrane filter (Jinteng, Tianjin, China).

The concentrations of raspberry ketone and $p$-coumaric acid were measured by high-performance liquid chromatography (HPLC, LC 20A LabSolutions, Shimadzu Corp., Kyoto, Japan) with an Agilent ExtendC18 $3.5 \mu \mathrm{m}$ column $(4.6 \times 250 \mathrm{~mm})$. The column was at $35{ }^{\circ} \mathrm{C}$, and a flow rate of $0.5 \mathrm{ml} / \mathrm{min}$ was used. Mobile phase A (65\%) was water with $0.1 \%(\mathrm{v} / \mathrm{v})$ formic acid; mobile phase B (35\%) was acetonitrile. Raspberry ketone and $p$-coumaric acid were detected via DAD detection. The raspberry ketone was detected at $222 \mathrm{~nm}, p$-coumaric acid was detected at $305 \mathrm{~nm}$.

\section{Abbreviations}

RK: Raspberry ketone; FAs: Fatty acids; 4CL: 4-Coumarate-CoA ligase; BAS: Benzalacetone synthase; RZS: Raspberry ketone reductase; CM: Complex enriched medium; MCM: Modified complex enriched medium; TCA: Tricarboxylic acid; LB: Luria-Bertani.

\section{Supplementary Information}

The online version contains supplementary material available at https://doi. org/10.1186/s12934-021-01551-0.

Additional file 1: Figure S1. The relative GFP intensity of the Pfrd3 promoter at different OD conditions. Figure S2. OD optimization and rotation speed optimization. (A) Production comparison between different OD conditions. (B) Production comparison between different rotation speeds. Figure S3. Remaining soybean oil of Fed-batch fermentation in 1-L bioreactors for RK production.

Additional file 2: Table S1. The primers used in this study.

\section{Acknowledgements}

The authors gratefully acknowledge funding from the following organizations: National Key Research and Development Project of China (Grant No. 2018YFA0901400); and National Natural Science Foundation of China (Grant No. 32070068).

\section{Authors' contributions}

YT and YB participated in the conception and design of the study and revised the manuscript. WL participated in data collection and analysis, and drafted the manuscript. CC and BL participated in original experiments, data collection and analysis. All authors read and approved the final manuscript.

\section{Funding}

National Key Research and Development Project of China (Grant No. 2018YFA0901400); National Natural Science Foundation of China (Grant No. 32070068).

\section{Availability of data and materials}

Not applicable.

\section{Declarations}

Ethics approval and consent to participate

Not applicable.

\section{Consent for publication}

Not applicable.

\section{Competing interests}

The authors declare that they have no competing interests.

\section{Author details}

${ }^{1}$ College of Forestry, Northeast Forestry University, No. 26 Hexing Road, Harbin, Heilongjiang Province 150040, PR China. ${ }^{2}$ CAS Key Laboratory of Microbial Physiological and Metabolic Engineering, State Key Laboratory of Microbial Resources, Institute of Microbiology, Chinese Academy of Sciences, NO. 1 Beichen West Road, Chaoyang District, Beijing 100101, PR China. ${ }^{3}$ University of Chinese Academy of Sciences, Shijingshan District, NO. 19A Yuquan Road, Beijing 100049, PR China. ${ }^{4}$ Heilongjiang Key Laboratory of Forest Food Resources Utilization, No. 26 Hexing Road, Harbin, Heilongjiang Province 150040, PR China.

Received: 16 November 2020 Accepted: 21 February 2021

Published online: 12 March 2021

\section{References}

1. Erickson B. Nelson, Winters P: Perspective on opportunities in industrial biotechnology in renewable chemicals. Biotechnol J. 2012;7:176-85.

2. Du J, Shao Z, Zhao H. Engineering microbial factories for synthesis of value-added products. J Ind Microbiol Biotechnol. 2011;38:873-90.

3. Gustavsson M, Lee SY. Prospects of microbial cell factories developed through systems metabolic engineering. Microb Biotechnol. 2016;9:610-7.

4. Nielsen J, Keasling Jay D. Engineering cellular metabolism. Cell. 2016;164:1185-97.

5. Vogt T. Phenylpropanoid biosynthesis. Mol Plant. 2010;3:2-20.

6. Ferrer JL, Austin MB, Stewart C, Noel JP. Structure and function of enzymes involved in the biosynthesis of phenylpropanoids. Plant Physiol Biochem. 2008;46:356-70.

7. Korkina LG. Phenylpropanoids as naturally occurring antioxidants: from plant defense to human health. Cell Mol Biol. 2007;53:15-25.

8. Ververidis F, Trantas E, Douglas C, Vollmer G, Kretzschmar G, Panopoulos N. Biotechnology of flavonoids and other phenylpropanoid-derived natural products. Part I: Chemical diversity, impacts on plant biology and human health. Biotechnol J. 2007;2:1214-34.

9. Tatsis EC, O'Connor SE. New developments in engineering plant metabolic pathways. Curr Opin Biotechnol. 2016;42:126-32.

10. Wu S, Chappell J. Metabolic engineering of natural products in plants; tools of the trade and challenges for the future. Curr Opin Biotechnol. 2008;19:145-52.

11. Brey LF, Włodarczyk AJ, Bang Thøfner JF, Burow M, Crocoll C, Nielsen I, Zygadlo Nielsen AJ, Jensen PE. Metabolic engineering of Synechocystis sp. PCC 6803 for the production of aromatic amino acids and derived phenylpropanoids. Metab Eng. 2020;57:129-39.

12. Liu Y, Nielsen J. Recent trends in metabolic engineering of microbial chemical factories. Curr Opin Biotechnol. 2019;60:188-97.

13. Palmer CM, Miller KK, Nguyen A, Alper HS. Engineering 4-coumaroylCoA derived polyketide production in Yarrowia lipolytica through a B-oxidation mediated strategy. Metab Eng. 2020;57:174-81.

14. Wang S, Zhang S, Xiao A, Rasmussen M, Skidmore C, Zhan J. Metabolic engineering of Escherichia coli for the biosynthesis of various phenylpropanoid derivatives. Metab Eng. 2015;29:153-9.

15. Xiong D, Lu S, Wu J, Liang C, Wang W, Wang W, Jin J-M, Tang S-Y. Improving key enzyme activity in phenylpropanoid pathway with a designed biosensor. Metab Eng. 2017:40:115-23.

16. Kiewning H. Structure and evolution of 4-coumarate:coenzyme A ligase (4CL) gene families. Biol Chem. 2001;382:645-54.

17. Kaneko M, Hwang El, Ohnishi Y, Horinouchi S. Heterologous production of flavanones in Escherichia coli: potential for combinatorial biosynthesis of flavonoids in bacteria. J Ind Microbiol Biotechnol. 2003;30:456-61.

18. Santos CN, Koffas M, Stephanopoulos G. Optimization of a heterologous pathway for the production of flavonoids from glucose. Metab Eng. 2011;13:392-400

19. Leonard E, Yan Y, Fowler ZL, Li Z, Lim CG, Lim KH, Koffas MA. Strain improvement of recombinant Escherichia coli for efficient production of plant flavonoids. Mol Pharm. 2008;5:257-65.

20. Braga A, Faria N. Bioprocess optimization for the production of aromatic compounds with metabolically engineered hosts: recent developments and future challenges. Front Bioeng Biotechnol. 2020;8:96. 
21. Wang C, Zheng P, Chen P. Construction of synthetic pathways for raspberry ketone production in engineered Escherichia coli. Appl Microbiol Biotechnol. 2019;103:3715-25.

22. Kang S-Y, Choi O, Lee JK, Hwang BY, Uhm T-B, Hong Y-S. Artificial biosynthesis of phenylpropanoic acids in a tyrosine overproducing Escherichia coli strain. Microb Cell Fact. 2012;11:153.

23. Hernández-Chávez G, Martinez A, Gosset G. Metabolic engineering strategies for caffeic acid production in Escherichia coli. Electron J Biotechnol. 2019:38:19-26.

24. Dellomonaco C, Clomburg JM, Miller EN, Gonzalez R. Engineered reversal of the $\beta$-oxidation cycle for the synthesis of fuels and chemicals. Nature. 2011:476:355-9.

25. Handke P, Lynch SA, Gill RT. Application and engineering of fatty acid biosynthesis in Escherichia coli for advanced fuels and chemicals. Metab Eng. 2011;13:28-37

26. Mannu A, Ferro M, Pietro MED, Mele A. Innovative applications of waste cooking oil as raw material. Sci Prog. 2019;102:153-60.

27. Mahlia TMI, Ismail N, Hossain N, Silitonga AS, Shamsuddin AH. Palm oil and its wastes as bioenergy sources: a comprehensive review. Environ Sci Pollut Res. 2019:26:14849-66.

28. Liu B, Xiang S, Zhao G, Wang B, Ma Y, Liu W, Tao Y. Efficient production of 3-hydroxypropionate from fatty acids feedstock in Escherichia coli. Metab Eng. 2019;51:121-30.

29. Liu N, Liu B, Wang G, Soong YHV, Tao Y, Liu W, Xie D. Lycopene production from glucose, fatty acid and waste cooking oil by metabolically engineered Escherichia coli. Biochem Eng J. 2020;155:107488.

30. Campbell JW, Cronan JE Jr. Escherichia coli FadR positively regulates transcription of the fabB fatty acid biosynthetic gene. J Bacteriol. 2001;183:5982-90.

31. Dirusso CC, Black PN. Bacterial long chain fatty acid transport: gateway to a fatty acid-responsive signaling system. J Biol Chem. 2004:279:49563-6.

32. Azizan A, Sherin D, DiRusso CC, Black PN. Energetics underlying the process of long-chain fatty acid transport. Arch Biochem Biophys. 1999;365:299-306.

33. Jeon E, Lee S, Lee S, Han SO, Yoon YJ, Lee J. Improved production of long-chain fatty acid in Escherichia coli by an engineering elongation cycle during fatty acid synthesis (FAS) through genetic manipulation. J Microbiol Biotechnol. 2012;22:990-9.

34. Kunau W-H, Dommes V, Schulz H. $\beta$-Oxidation of fatty acids in mitochondria, peroxisomes, and bacteria: a century of continued progress. Prog Lipid Res. 1995:34:267-342.

35. Watts KT, Lee PC, Schmidt-Dannert C. Biosynthesis of plant-specific stilbene polyketides in metabolically engineered Escherichia coli. BMC Biotechnol. 2006;6:22-22.

36. Milke L, Marienhagen J. Engineering intracellular malonyl-CoA availability in microbial hosts and its impact on polyketide and fatty acid synthesis. Appl Microbiol Biotechnol. 2020;104:6057-65.

37. Palmer CM, Alper HS. Expanding the chemical palette of industrial microbes: metabolic engineering for type III PKS-derived polyketides. Biotechnol J. 2019;14:e1700463.

38. Ehlting J, Shin JJ, Douglas CJ. Identification of 4-coumarate: coenzyme A ligase (4CL) substrate recognition domains. Plant J. 2001;27:455-65.

39. Koeduka T, Watanabe B, Suzuki S, Hiratake J, Mano J, Yazaki K. Characterization of raspberry ketone/zingerone synthase, catalyzing the alpha, beta-hydrogenation of phenylbutenones in raspberry fruits. Biochem Biophys Res Commun. 2011;412:104-8.

40. Lim CG, Fowler ZL, Hueller T, Schaffer S, Koffas MA. High-yield resveratrol production in engineered Escherichia coli. Appl Environ Microbiol. 2011;77:3451-60

41. Baba T, Ara T, Hasegawa M, Takai Y, Okumura Y, Baba M, Datsenko KA, Tomita M, Wanner BL, Mori H. Construction of Escherichia coli K-12 inframe, single-gene knockout mutants: the Keio collection. Mol Syst Biol. 2006;2006(2):0008

42. Datsenko KA, Wanner BL. One-step inactivation of chromosomal genes in Escherichia coli K-12 using PCR products. Proc Natl Acad Sci USA. 2000;97:6640-5.

\section{Publisher's Note}

Springer Nature remains neutral with regard to jurisdictional claims in published maps and institutional affiliations.
Ready to submit your research? Choose BMC and benefit from:

- fast, convenient online submission

- thorough peer review by experienced researchers in your field

- rapid publication on acceptance

- support for research data, including large and complex data types

- gold Open Access which fosters wider collaboration and increased citations

- maximum visibility for your research: over $100 \mathrm{M}$ website views per year

At $\mathrm{BMC}$, research is always in progress.

Learn more biomedcentral.com/submissions 